\title{
Simple concentration method enables the use of gargle and mouthwash instead of nasopharyngeal swab sampling for the diagnosis of COVID-19 by PCR
}

\author{
Tanil Kocagoz ${ }^{1,2} \oplus \cdot$ Ozge Can $^{3} \cdot$ Neval Yurttutan Uyar ${ }^{1,4} \cdot$ Ece Aksoy $^{2} \cdot$ Tuba Polat $^{2} \cdot$ Dilara Cankaya $^{2} \cdot$ Betul Karakus $^{2}$. \\ Erkan Mozioglu ${ }^{2} \cdot$ Sesin Kocagoz ${ }^{5}$
}

Received: 6 May 2021 / Accepted: 26 July 2021 / Published online: 26 August 2021

(c) The Author(s), under exclusive licence to Springer-Verlag GmbH Germany, part of Springer Nature 2021

\begin{abstract}
Since its emergence in December 2019, SARS-CoV-2 is causing one of the most devastating pandemics in human history. Currently, the most important method for definitive diagnosis of COVID-19 is identification of SARS-CoV-2 RNA in nasopharyngeal swab samples by RT-PCR. Nasopharyngeal swab sampling is a discomforting procedure sometimes with adverse effects, which also poses a risk for infection for the personnel performing the sampling. We have developed a new method for concentrating biological samples, which enabled us to use gargle and mouthwash samples to be used in RT-PCR, for the diagnosis of COVID-19, as an alternative to nasopharyngeal swab samples. We have analyzed nasopharyngeal and gargle and mouthwash samples, before and after concentration, of 363 patients by RT-PCR for the presence of SARS-CoV-2. Among 114 patients in which SARS-CoV-2 was identified in at least one of their samples, the virus was identified in 76 (66.7\%), 67 $(58.8 \%)$, and $101(88.6 \%)$ of nasopharyngeal swab, gargle, and mouthwash samples before and after concentration, respectively. When concentrated by our new method, gargle and mouthwash samples can be used instead of nasopharyngeal samples in identification of SARS-CoV-2 by RT-PCR, with the same or better sensitivity. Eliminating the need for nasopharyngeal sampling will save the patients from an invasive and painful procedure and will lower the risk of infection for the healthcare personnel taking the sample. This easy sampling procedure may decrease the workload of hospitals, shorten the turnaround time of obtaining test results, and thus enable rapid isolation of infected patients.
\end{abstract}

Keywords COVID-19 $\cdot$ SARS-CoV-2 $\cdot$ PCR $\cdot$ Mouthwash $\cdot$ Virus concentration $\cdot$ Microorganism concentration

Tanil Kocagoz and Ozge Can have contributed equally to this work.

Tanil Kocagoz

tanilkocagoz@gmail.com

Ozge Can

ozge.can@acibadem.edu.tr

1 School of Medicine, Department of Medical Microbiology, Acibadem University, Istanbul, Turkey

2 Department of Medical Biotechnology, Institute of Health Sciences, Acibadem University, Istanbul, Turkey

3 Faculty of Engineering, Department of Medical Engineering, Acibadem University, Istanbul, Turkey

4 Acibadem Labmed, Istanbul, Turkey

5 Department of Infectious Diseases, School of Medicine, Acibadem University, Istanbul, Turkey

\section{Introduction}

Severe acute respiratory syndrome (SARS-CoV-2) is a positive-sense and single-stranded RNA (ssRNA) virus [1]. Since its emergence in December 2019, SARS-CoV-2 is continuing to cause one of the most devastating pandemics in human history. COVID-19 led the entire world to face an economic crisis, which additionally makes it harder to control the pandemic. Although several vaccines are now in application, it is estimated that it will take at least until the end of 2021 to control the pandemic. Until majority of the world population develops immunity by effective vaccination or by being infected, protective masks, social distancing, and quarantine rules will continue to be the most important measures to slow down the speed of transmission in order to stop overwhelming the health systems [2].

Rapid diagnosis and isolation of people carrying SARSCoV-2 before they transmit the virus to uninfected people 
are the key to break the chain of transmission. Currently, the most important diagnostic method for definitive diagnosis of COVID-19 is identification of SARS-CoV-2 RNA in nasopharyngeal swab samples by RT-PCR [3, 4]. Nasopharyngeal swab sampling is a painful process for patients, sometimes leading to serious complications. It requires trained personnel and poses a risk of infection for the person who does the sampling [5-7]. It is a time-consuming application which created waiting lines in front of the testing centers in many parts of the world, by the people who wait for hours to give a nasopharyngeal swab sample. In this study, we have investigated the possibility of using gargle and mouthwash samples, after concentrating them by a new product named MyMagiCon ${ }^{\circledR}$ (GigaBioMol, Bio-T, Istanbul, Turkey) that we have recently developed, for the diagnosis of COVID-19, as an alternative to nasopharyngeal swab sampling.

MyMagiCon ${ }^{\circledR}$ is a powder mixture that contains a special polymer that removes small molecules quickly from solutions. The elastic polymer beads swell quickly by absorbing water and other small molecules, concentrating microorganisms, and macromolecules. MyMag$i C o n-R W 100 \AA$ is intended for concentrating gargle and mouthwash, for the diagnosis of infectious agents like SARS-CoV-2, Influenza virus, and other agents causing infection in the respiratory system. Microorganisms are concentrated if they are in intact form. However, even if the organisms are lysed and their nucleic acids and antigens are released in the solution, these will be also concentrated, since molecules larger than approximately $0.5 \mathrm{kD}$ will stay outside the polymer beads while small molecules that can penetrate the pores of polymer meshes will be removed. Thus, MyMagiCon-RW100® concentrates the microorganisms and their macromolecules 10 to 20 times.

\section{Methods}

\section{Stability of SARS-CoV-2 in mouthwash samples}

To evaluate gargle and mouthwash as an alternative to nasopharyngeal swab samples for the diagnosis of COVID-19, we first evaluated the stability of SARS-CoV-2 in mouthwash samples. For this purpose, mouthwash samples were collected from 10 healthy volunteers. From five of these samples, $1 \mathrm{~mL}$ aliquots was spiked with SARS-CoV-2, grown in cell culture and inactivated, to a final concentration of $10^{15}$ and the other five with $10^{14}$ copies $/ \mathrm{mL}$. Each aliquot was split into two equal parts in micro-centrifuge tubes and one set of these were kept at room temperature while the other set at $4{ }^{\circ} \mathrm{C}$. The concentration of virus in each sample was quantified by RT-PCR using a commercial kit (Bioeksen, Istanbul, Turkey) at days $0,3,5,7$, and 10 . The average copy number in samples stored at room temperature and $4{ }^{\circ} \mathrm{C}$ was calculated for each of these days, and the change in copy number of viruses was determined (Fig. 1).

\section{Determination of the sensitivity of RT-PCR in identification of SARS-CoV-2 in nasopharyngeal and concentrated mouthwash samples}

A total of 363 volunteers above the age of 18 , who were admitted to the Acibadem Altunizade Hospital (Istanbul) by symptoms of respiratory infection, were included in the study. After collecting nasopharyngeal swab samples, patients were instructed to take a few sips of regular drinking water, and then to gargle and rigorously rinse their mouth forcefully with this water for at least $10 \mathrm{~s}$ and put it back to an empty cup. (Ethical approval for the study was obtained from the Acibadem University Ethical Committee, ATADEK approval No: 2020-14/2.)
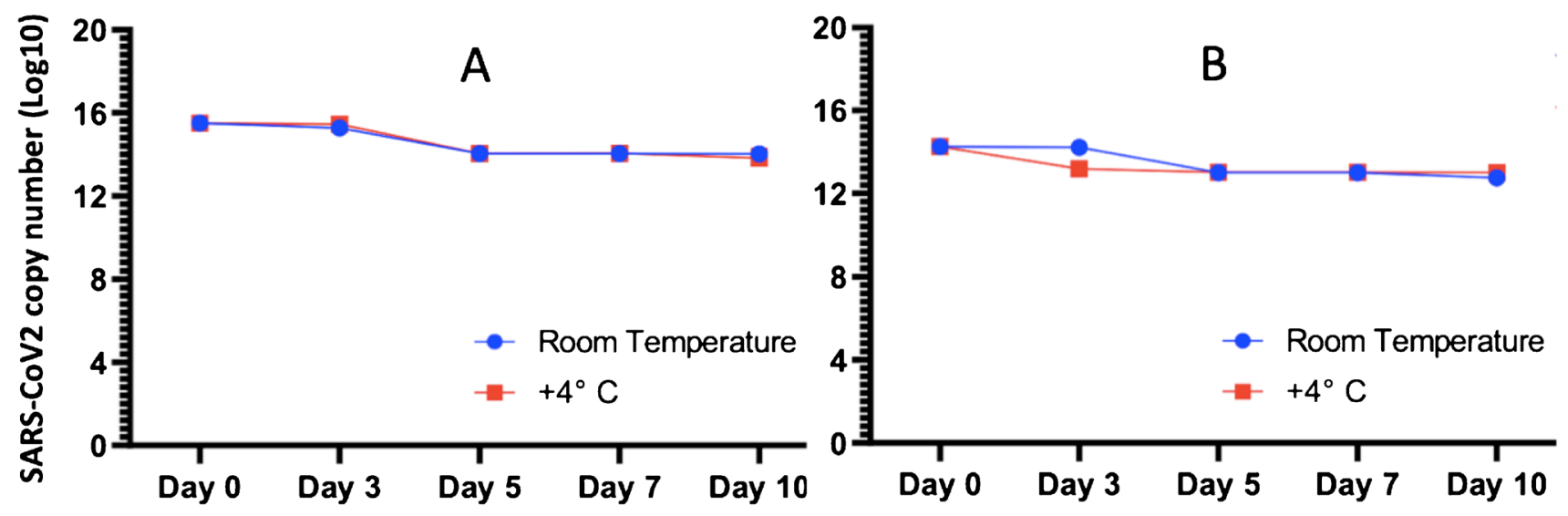

Fig. 1 The stability of SARS-CoV-2 RNA in mouthwash samples at room temperature and $4{ }^{\circ} \mathrm{C}$. The starting number of RNA copies in samples shown in graph $\mathbf{A}$ was $10^{15} / \mathrm{mL}$ and in samples shown in graph $\mathbf{B}$ was $10^{14} / \mathrm{mL}$ 
Gargle and mouthwash samples were concentrated using MyMagiCon- $R W 100 \AA$ as instructed in the user guide. Briefly, $20 \mathrm{~mL}$ of sample was put into the tube and waited for 5 min for the absorbent beads to swell and absorb most of the fluid, and the mixture turned in a gel-like form. The concentrated sample was collected with an automatic pipette by inserting the pipette tip in between the beads and aspirating the fluid. MyMagiCon $\AA$ powder is a polymer mesh that absorbs water and other small molecules with a molecular weight approximately less than $0.5 \mathrm{kD}$. The concentration of SARS-CoV-2 in gargle and mouthwash takes about $5 \mathrm{~min}$.

Nasopharyngeal swab sample, gargle and mouthwash before and after concentration, was analyzed for the presence of SARS-CoV-2 by direct RT-PCR, without RNA extraction, using commercial PCR kits (Bioeksen and A1 Lifesciences, Istanbul, Turkey). Every batch of samples studied by PCR included negative and positive controls to eliminate falsepositive and false-negative results.

\section{Statistical analysis}

Pearson chi-square test has been performed to 363 samples' three nominal measurement levels: mouthwash (MW), mouthwash concentrated with MyMagiCon ${ }^{\circledR}$ (MMCMW), and nasopharyngeal swap sample (NPS) (IBM Corp. Released 2017. IBM SPSS Statistics for Windows, version 25.0. Armonk, NY: IBM Corp.). In this case, the independent variable was the testing method for COVID-19 diagnosis and the dependent variable was the test result with two levels: negative and positive (degree of freedom $=1$ ).

\section{Results}

The stability of SARS-CoV-2 RNA in mouthwash samples, stored at room temperature and $4{ }^{\circ} \mathrm{C}$, is shown in Fig. 1 . There was no significant change in the copy number of SARS-CoV-2 RNA after 3 days of storage at both room temperature and $4{ }^{\circ} \mathrm{C}$. There was about one log decrease after storing 10 days at both of these temperatures. Storing mouthwash samples either at room temperature or $4{ }^{\circ} \mathrm{C}$ showed no significant difference in the stability of viral RNA.

In 114 samples, SARS-CoV2 RNA was identified in at least one of the three sample types. The viral RNA was detected in $76(66.7 \%)$ nasopharyngeal, in $67(58.8 \%)$ gargle and mouthwash before concentration, and in 101 (88.6\%) after concentration among the total RT-PCR-positive patients (Fig. 2). In 10 patients, nasopharyngeal swab samples, which were positive for SARS-CoV-2, were negative in their concentrated gargle and mouthwash samples. On the other hand, SARS-Cov-2 was positive in 35 concentrated gargle and mouthwash samples, which were negative in nasopharyngeal samples of the same patients.

Concentration of samples increased the number of samples in which SARS-CoV-2 RNA was detected by $29.8 \%$. The effect of concentration on the detection of SARSCoV-2 RNA is shown in Fig. 3. In samples having high concentration of virus, compared to the original sample, the amplification curve passed the cutoff threshold $(\mathrm{Ct})$ value several cycles earlier after concentration by MyMagiCon ${ }^{\circledR}$ (Fig. 3A). In samples, which contained the virus at
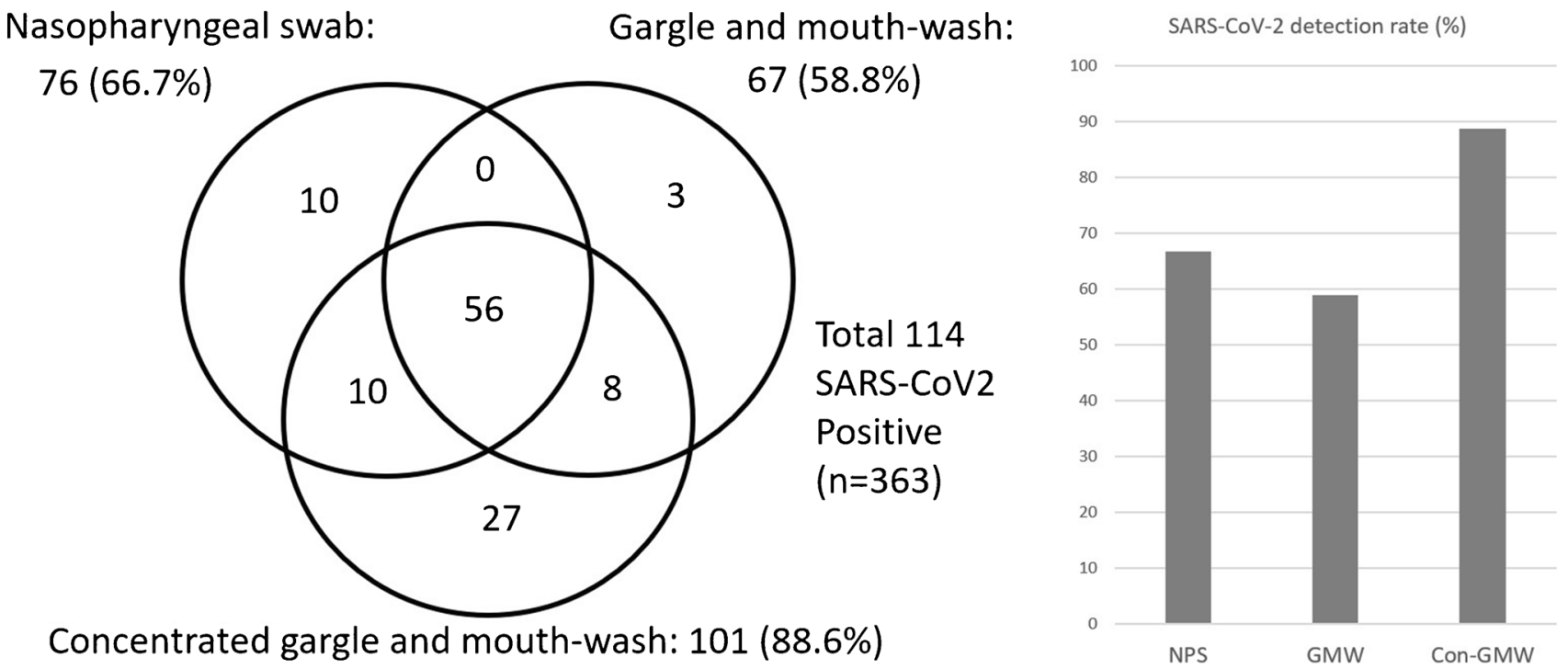

Fig. 2 Detection of SARS-CoV-2 RNA, by RT-PCR in nasopharyngeal swab samples, in gargle and mouthwash samples, before and after concentration by MyMagiCon-RW100® 


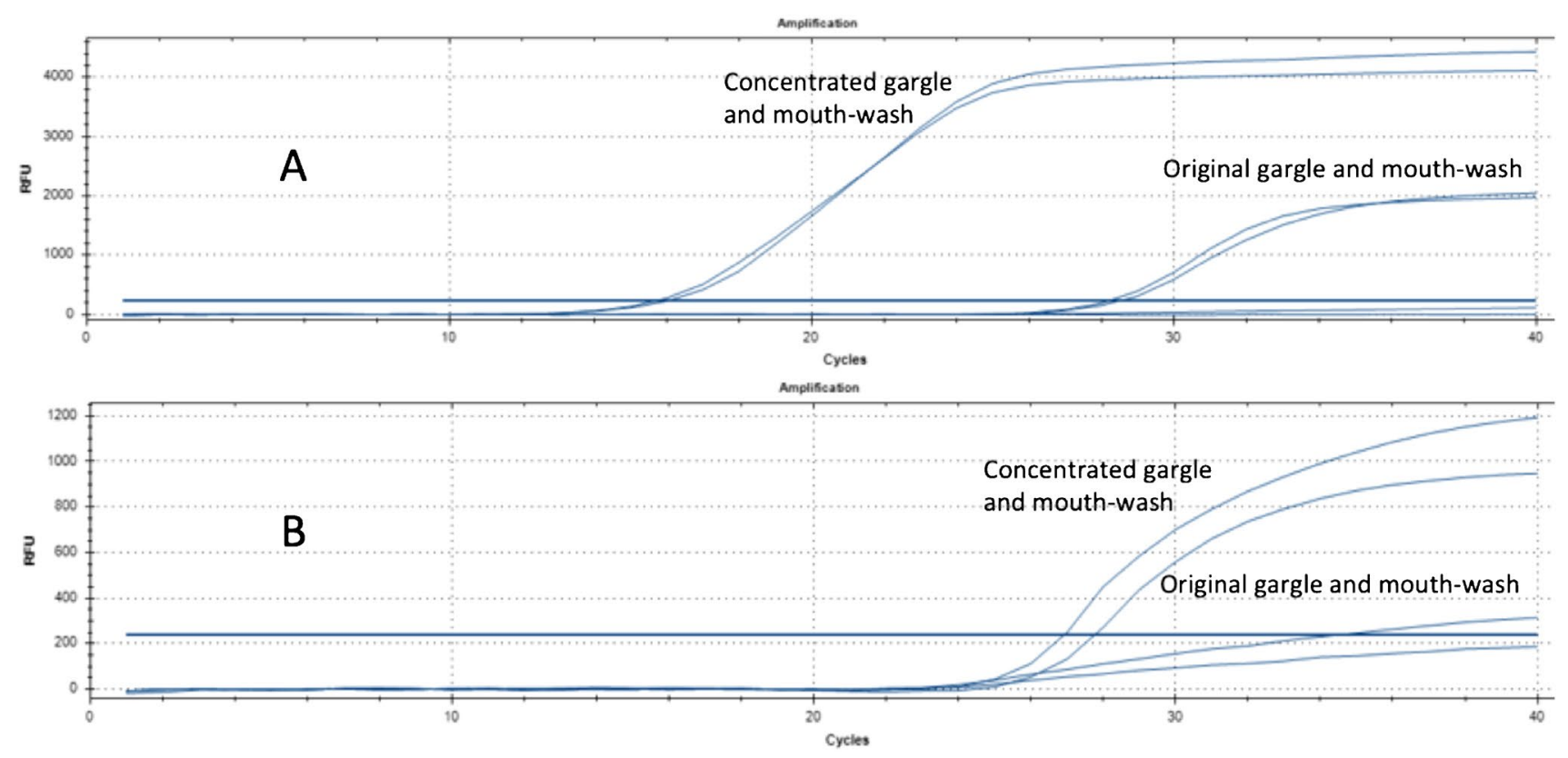

Fig. 3 The effect of concentration of gargle and mouthwash samples on the detection limit of SARS-CoV-2 by RT-PCR. Each sample was evaluated in duplicate. In graph $\mathbf{A}$, it was possible to detect SARS-

a concentration of below the limit of detection, it became possible to detect the virus after concentration (Fig. 3B).

The proportion of NPS and MMC-MW samples was significantly different by diagnosis $(X 2(1, N=363)=3.841$, $p=0.00007, p>0.05$, Pearson chi-square 187.51. The minimum expected count is 18.64). MW and NPS crosstabulation results indicated that there was a statistically significant difference between two test methods (X2 (1, $N=363)=3.841, p=0.007, p>0.05$, Pearson chi-square: 166.72. 0 cells $(0.0 \%)$ have expected count less than 5 . The minimum expected count is 21.15). Similarly, MW and MMC-MW cross-tabulation results stated significant difference between two test methods $(X 2(1, N=363)=3.841$, $p=0.003, p>0.05$; Pearson chi-square: 194.79. 0 cells $(0.0 \%)$ have expected count less than 5 . The minimum expected count is 14.03).

\section{Discussion}

COVID-19 became one of the most devastating pandemics in human history. As in all pandemic diseases, rapid diagnosis of people carrying the infectious agent and their quarantine before they transmit the disease to healthy people are the key measure to control the disease in large populations [2]. Currently, PCR is the main diagnostic tool for rapid and sensitive diagnosis of COVID-19 [3, 4].

The gold standard test for detecting SARS-CoV-2 is considered to be the analysis by RT-PCR of a sample obtained
CoV-2 without concentration at later cycles; however, the detection of the virus was not possible without concentration in the example shown in graph $\mathbf{B}$

by nasopharyngeal swab. Therefore, the most widely used application for obtaining samples for PCR is by a nasopharyngeal swab. However, besides being very discomforting, many adverse effects associated with nasopharyngeal swab sampling, including epistaxis in $8.3 \%$ of cases, have been reported. Additionally, nasopharyngeal swab sampling poses an important risk of transmission of the virus to healthcare personnel who have to do tens to hundreds of sampling every day, in centers with high admission rate [5-7].

It is expected to find SARS-CoV-2 in the oral secretions of COVID-19 patients. Epithelial cells in the oral cavity have been shown to express large amount of ACE2 receptors, which plays a key role in the entry and replication of SARSCoV-2 [8]. Nasopharynx and oropharynx are not separated from each other physically and it is logical to think that the secretions in the nasopharynx will be mixed into the oral secretions. Additionally, virus particles in the blood may pass into exudates produced in the oral cavity.

Several studies revealed the presence of SARS-CoV-2 in saliva of COVID-19 patients. The sensitivity of RT-PCR analysis of saliva specimens was 66 to $92 \%$ for COVID-19 as compared with the standard diagnosis with nasopharyngeal swabs $[9,10]$. In a recent study, Goldfarb et al. compared self-collected saline gargle samples as an alternative to healthcare worker-collected nasopharyngeal swabs, for COVID-19 diagnosis. They have found that mouthwash and gargle samples were significantly more likely to be positive than saliva and nasopharyngeal swab samples. They have 
also shown that the stability of SARS-CoV-2 RNA is much better in gargle and mouthwash samples than nasopharyngeal swab samples in especially long-term storage (11). In previous studies, it has been shown that gargle sampling resulted in better performance in terms of sensitivity than throat sampling in the detection of other respiratory pathogens (12).

In this study, there was not any loss of viral RNA after storing the mouthwash samples for 3 days at room temperature and only tenfold decrease at the end of 10 days. Storing at $4{ }^{\circ} \mathrm{C}$ preserved the viral RNA as well as room temperature but it did not improve the quantity of viral RNA that can be detected by RT-PCR. Inspired by these data, we investigated the possibility of using concentrated gargle and mouthwash samples instead of nasopharyngeal swab samples with the same or better efficiency in the diagnosis of COVID-19.

Recently, we have developed a method for concentrating biological fluids by the help of elastic polymer meshes that absorb water and other molecules and thus concentrate microorganisms and macromolecules. In a recent study, we have shown that, by this method, it was possible to increase the sensitivity of antigen tests detecting tuberculosis antigens in urine samples of tuberculosis patients (unpublished internal data). In this study, we have evaluated the efficiency of MyMagiCon-RW100®, which is intended for concentrating gargle and mouthwash.

The results of this study showed that gargle and mouthwash samples can be used as efficiently as nasopharyngeal swab samples, after concentrating by MyMagiCon-RW100®. Among all 114 patients in whom SARS-CoV-2 RNA was identified by RT-PCR in at least one of the nasopharyngeal or gargle and mouthwash samples, $76(66.7 \%)$ was identified in nasopharyngeal swab and $67(58.8 \%)$ in gargle and mouthwash samples. However, when gargle and mouthwash samples were concentrated by MyMagiCon-RW100®, it was possible to identify SARS-CoV-2 RNA in 101 (88.6\%) of samples making them better samples than nasopharyngeal samples for the diagnosis of COVID-19. An interesting finding was the presence of SARS-CoV-2 RNA in only nasopharyngeal samples of 10 patients and in only concentrated gargle and mouthwash samples of 35 patients. Since negative controls were negative in RT-PCR when studying these 35 gargle and mouthwash samples, we believe that these are not false-positive results. If the virus was really present in only nasopharyngeal or oropharyngeal cavity at the time of sampling, or there was a problem with collecting the samples properly, needs further investigation. When the sensitivity of a newly developed method is better than the gold standard method, it is not possible to determine the sensitivity unless a third method with better sensitivity or data about clinical findings of the patients are available. This was not possible in this study since there is currently no diagnostic method with better sensitivity than PCR and it was not possible to reach to the clinical follow-up data of the patients.

Although gargle and mouthwash sampling is non-invasive and much easier to collect, this approach can only be used if the person being tested is able to gargle. Patients from whom a gargle cannot be obtained (dysphagia, dementia, or infants) should be swabbed. Using gargle and mouthwash samples instead of nasopharyngeal swab samples will increase patient compliance, eliminate the adverse effects of nasopharyngeal swab sampling, significantly decrease the infection risk of health personnel obtaining the samples, and prominently lower the workload of healthcare centers. When the rapid antigen tests with sensitivities close to RT-PCR become available, MyMagiCon-RW100® may enable rapid diagnosis from mouthwash samples which may be applied in hospitals or even at homes for self-testing. For this purpose, further studies investigating the sensitivity of rapid antigen tests in concentrated gargle and mouthwash samples, compared to nasopharyngeal swab samples, are needed.

Acknowledgements The development of MyMagiCon ${ }^{\circledR}$ was supported by Foundation for Innovative New Diagnostics (FIND) originally for identification of tuberculosis antigens in urine. We are grateful to Emmanuel Moreau, Kavi Ramjeet, and Morten Ruhwald from FIND, for their guidance for the development of this product and FIND for financial support. We would like to thank Ercument Ovali, Cihan Tastan, and Derya Kancagi for providing inactivated SARS-CoV-2. We would also like to thank our technicians Gorkem Gun, Selma Cat, Yesim Gun, and Acibadem University Medical interns, Cansu Buke, Bayram Kara, Milena Can, and Melis Ureksoy for obtaining and processing the clinical samples.

\section{Declarations}

Competing interests The authors Tanil Kocagoz and Ozge Can are the founders of GigaBioMol and scientific advisors of Bio-T.

Ethical approval Ethical approval for the study was obtained from Acibadem University Ethical Committee, ATADEK approval No: $2020-14 / 2$.

Consent to participate Volunteers provided signed informed consent.

Consent for publication All authors give their consent for the publication of this manuscript.

\section{References}

1. Siddell SG, Ziebuhr J, Snijder EJ (2010) Coronaviruses, toroviruses, and arteriviruses. In: Mahy BW, Meulen VT, Borriello SP, Murray PR, Funke G, Kaufmann SH, Steward MW, Merz WG, Hay RJ, Cox F, Wakelin D, Gillespie SH, Despommier DD, Borriello SP, Murray PR, Funke G (eds) Topley \& Wilson's microbiology and microbial infections. https://doi.org/10.1002/97804 70688618.taw0245 
2. Nicola M, Alsafi Z, Sohrabi C, Kerwan A, Al-Jabir A, Iosifidis C, Agha M, Agha R (2020) The socio-economic implications of the coronavirus pandemic (COVID-19): a review. Int J Surg 78:185-193

3. Tang YW, Schmitz JE, Persing DH, Stratton CW (2020) Laboratory diagnosis of COVID-19: current issues and challenges. J Clin Microbiol 58:1-22

4. Afzal A (2020) Molecular diagnostic technologies for COVID-19: limitations and challenges. J Adv Res 26:149-159

5. Gopaul R, Davis J, Gangai L, Goetz L (2020) Practical diagnostic accuracy of nasopharyngeal swab testing for novel coronavirus disease 2019 (COVID-19). West J Emerg Med. https://doi.org/ 10.5811/westjem.2020.8.48420:1-4

6. Gupta K, Bellino P, Charness ME (2020) Adverse effects of nasopharyngeal swabs: 3-D printed versus commercial swabs. Infect Control Hosp Epidemiol 5594:4-7

7. Mughal Z, Luff E, Okonkwo O, Hall CEJ (2020) Test, test, test-a complication of testing for coronavirus disease 2019 with nasal swabs. J Laryngol Otol 134:646-649

8. Xu K, Chen Y, Yuan J, Yi P, Ding C, Wu W, Li Y, Ni Q, Zou R, Li X, Xu M, Zhang Y, Zhao H, Zhang X, Yu L, Su J, Lang G, Liu J, Wu X, Guo Y, Tao J, Shi D, Yu L, Cao Q, Ruan B, Liu L, Wang Z, Xu Y, Liu Y, Sheng J, Li L (2020) Factors associated with prolonged viral RNA shedding in patients with Coronavirus disease 2019. Clin Infect Dis 71:799-806
9. Azzi L, Carcano G, Gianfagna F, Grossi P, Gasperina DD, Genoni A, Fasano M, Sessa F, Tettamanti L, Carinci F, Maurino V, Rossi A, Tagliabue A, Baj A (2020) Saliva is a reliable tool to detect SARS-CoV-2. J Infect 81:e45-e50

10. Fernandes LL, Pacheco VB, Borges L, Athwal HK, de Paula EF, Bezinelli L, Correa L, Jimenez M, Dame-Teixeira N, Lombaert IMA, Heller D (2020) Saliva in the diagnosis of COVID-19: a review and new research directions. J Dent Res 99:1435-1443

11. Goldfarb DM, Tilley P, Al-Rawahi GN, Srigley JA, Ford G, Pedersen H, Pabbi A, Hannam-Clark S, Charles M, Dittrick M, Gadkar VJ, Pernica JM, Hoang LMN (2021) Self-collected saline gargle samples as an alternative to health care worker-collected nasopharyngeal swabs for COVID-19 diagnosis in outpatients. J Clin Microbiol 59(4):e02427-e2520. https://doi.org/10.1128/JCM. 02427-20.PMID:33514627;PMCID:PMC8092743

12. Bennett S, Davidson RS, Gunson RN (2017) Comparison of gargle samples and throat swab samples for the detection of respiratory pathogens. J Virol Methods 248:83-86. https://doi.org/10. 1016/j.jviromet.2017.06.010

Publisher's note Springer Nature remains neutral with regard to jurisdictional claims in published maps and institutional affiliations. 